\title{
BMJ Open Association of the neutrophil to lymphocyte ratio and clinical outcomes in patients with lung cancer receiving immunotherapy: a meta-analysis
}

To cite: Jin J, Yang L, Liu D, et al. Association of the neutrophil to lymphocyte ratio and clinical outcomes in patients with lung cancer receiving immunotherapy: a meta-analysis. BMJ Open 2020;10:e035031. doi:10.1136/ bmjopen-2019-035031

- Prepublication history and additional material for this paper are available online. To view these files, please visit the journal online (http://dx.doi. org/10.1136/bmjopen-2019035031).

Received 17 0ctober 2019 Revised 14 April 2020 Accepted 23 April 2020
Check for updates

(c) Author(s) (or their employer(s)) 2020. Re-use permitted under CC BY-NC. No commercial re-use. See rights and permissions. Published by BMJ.

Department of Respiratory \& Critical Care Medicine, West China Hospital, Sichuan University, Chengdu, China

Correspondence to

Dr Weimin Li;

weimin003@yahoo.com

\section{Abstract}

Objectives To explore the relationship between the pretreatment or post-treatment neutrophil to lymphocyte ratio (NLR) and overall survival (OS)/progression-free survival (PFS) in patients with lung cancer receiving immunotherapy.

Design We searched several databases to collect relevant studies conducted until July 2019 . We carefully reviewed the full text of the included publications and combined the HRs and $95 \%$ Cls to assess the association between the NLR and survival time in patients with lung cancer receiving immunotherapy.

Data sources PubMed, the Cochrane Library, Embase and Web of Science

Eligibility criteria Studies reporting the prognostic value of the NLR in patients with lung cancer receiving immunotherapy were enrolled.

Data extraction and synthesis Basic information on the articles and patients (NLR cut-off value, NLR at baseline and HRs with $95 \%$ Cls for OS and PFS) was extracted by two authors independently. The pooled HRs of OS and PFS were synthesised using the random effects or fixed effects model.

Results Twenty-three studies with 2068 patients were enrolled. Among all patients, 1305 (64.0\%) were men and $643(31.4 \%)$ were diagnosed with squamous cell carcinoma (SCC). In a pooled analysis of OS and PFS from all studies, an elevated NLR predicted poor OS $(\mathrm{HR}=1.62$; 95\% Cl: 1.41 to $1.87 ; p<0.001)$ and PFS ( $\mathrm{HR}=1.47$; $95 \% \mathrm{Cl}: 1.25$ to $1.72 ; p<0.001)$. Subgroup analyses stratified showed that the post-treatment NLR was not significantly related to $\mathrm{OS}$ and that patients in Asia had significantly higher HRs than those in Europe and America. Furthermore, the proportion of SCC and baseline NLR could affect the prognostic value of the NLR.

Conclusions Our study found that an elevated NLR was associated with poor OS and PFS in patients with lung cancer receiving immunotherapy and that several clinical factors might have an impact on the predictive value of the NLR in the survival of patients with lung cancer.

\section{INTRODUCTION}

Lung cancer is the most prevalent cancer and life-threatening malignancy worldwide. ${ }^{1}$ The pathogenesis of lung cancer is complicated, and the primary treatments for patients with
Strengths and limitations of this study

- Verification of the prognostic value of the neutrophil to lymphocyte ratio (NLR) in a large number of patients with lung cancer who received immunotherapy.

- Different clinical characteristics could affect the prognostic value of the NLR.

High heterogeneity was present in this analysis

lung cancer are surgery and chemotherapy. Unfortunately, most patients with lung cancer are diagnosed at advanced stages, and the benefits achieved from chemotherapy in advanced patients with lung cancer are relatively small. Recently, many studies have revealed that tumour cells can evade the antitumour responses of $\mathrm{T}$ cells by controlling the combined responses of programmed cell death protein 1 (PD-1) and programmed cell death ligand-1 (PD-L1). ${ }^{2}$ Nivolumab, pembrolizumab, atezolizumab, durvalumab, ipilimumab and tremelimumab have successfully changed clinical experiences in lung cancer treatment. ${ }^{3}$ Tumour mutational burden, ${ }^{4}$ neoantigens ${ }^{5}$ and classical monocytes in the peripheral blood ${ }^{6}$ and PD-L1 expression on tumour cells in particular ${ }^{7}$ are effective predictive biomarkers for immune checkpoint therapy in lung cancer. Systemic inflammation in patients with cancer is believed to influence the growth and migration of tumours via certain inflammatory factors. ${ }^{8}$ An elevated level of systemic inflammation, including Glasgow Prognostic Score (GPS), neutrophil to lymphocyte ratio (NLR), platelet to lymphocyte ratio (PLR) and C-reactive protein to albumin ratio, have been indicated to be associated with poor survival in patients with solid tumours. ${ }^{9-11}$ However, data on the prognostic value of the pretreatment NLR in patients with lung cancer receiving immunotherapy remain scarce and 
inconsistent. Therefore, we reviewed available publications and conducted a meta-analysis to explore the prognostic value of the pretreatment NLR for overall survival (OS) and progression-free survival (PFS) in clinical trials on patients with lung cancer receiving immunotherapy.

\section{MATERIALS AND METHODS}

\section{Patient and public involvement}

No patient was involved

\section{Search strategy}

The PRISMA guidelines for a systematic review and meta-analysis were strictly followed in this article (registration number PROSPERO: CRD42018104856). An online search was conducted to identify relevant publications in the PubMed, Cochrane Library, Web of Science and Embase databases. The following words were used to search for studies on the associations between the pretreatment NLR and survival time in patients with lung cancer published before July 2019: 'pulmonary neoplasms', 'neutrophil lymphocyte ratio', 'immunotherapy', 'programmed death receptor-1' and 'immune checkpoint inhibitor'. A full electronic search strategy is provided in the supplementary information (online supplementary table 1). Additional studies were selected for a full-text review were selected by exploring the references cited in the selected articles and relevant reviews. The articles were limited to the English language, but there were no restrictions on the minimum number of patients. Two authors (JJ and LY) independently reviewed the titles and abstracts of the retrieved articles to select the potentially relevant articles for a careful assessment.

\section{Eligibility criteria}

The inclusion criteria were as follows: (1) retrospective or prospective studies published before July 2019; (2) all patients enrolled in the studies were diagnosed with lung cancer by biopsy and received immunotherapy; (3) the value of the NLR was calculated based on the level of neutrophils and lymphocytes and (4) HRs and 95\% CIs were provided and data necessary to calculate them were reported.

The exclusion criteria were as follows: (1) review, meeting abstract, letter or full text unavailable in English; (2) non-human studies and (3) research that did not provide the value of the NLR.

\section{Data extraction}

From each study, the name of the study, first author, year of publication, study design, number of patients, sex distribution, age, median follow-up time, histology, NLR cut-off value, NLR at baseline, line of therapy, drugs and HRs with 95\% CIs for OS and PFS were extracted by two authors (DL and LY). If univariate and multivariate analysis results were simultaneously reported, only the multivariate analysis results were extracted. Any disagreements between the authors were resolved by a discussion and consensus. The most recent study was chosen when duplicate studies occurred.

\section{Quality assessment}

The primary studies were assessed by the Newcastle-Ottawa quality assessment Scale (NOS). The quality assessment was conducted by two independent researchers (JJ and DL). The studies in which the mark was between 6 and 9 points were regarded as high-quality studies.(http://www. ohri.ca/programs/clinical_epidemiology/oxford.asp)

\section{Statistical analysis}

The primary endpoints were the OS and PFS of patients with lung cancer receiving immunotherapy. PFS was defined as the time from the initial date of immunotherapy to the date of progression or death. OS was calculated from the date of inclusion to the time of death from any cause. HRs with $95 \%$ CIs were directly obtained from the articles or estimated from the Kaplan-Meier curves according to the methods reported by Tierney et al. ${ }^{12} \mathrm{We}$ calculated the pooled HRs of OS and PFS using random effects or fixed effects model. We performed the Q-test to assess between-study heterogeneity and calculated the $\mathrm{I}^{2}$ statistic, which expresses the percentage of the total observed variability due to study heterogeneity. The heterogeneity between studies was considered small if the $\mathrm{I}^{2}$ statistic was less than $50 \%$ and the $\mathrm{p}$ value for the Q-test was less than 0.05 . We performed a subgroup analysis to detect the source of heterogeneity. In addition, we considered only subgroups that included more than two studies. Publication bias was assessed by Egger's and Begg's test, and significant publication bias was defined as a $\mathrm{p}<0.10 .{ }^{13}$ The trim and fill method was applied when significant publication bias was found to confirm the pooled results. Sensitivity analyses were carried out by excluding each study individually from the meta-analysis. ${ }^{14}$ All statistical analyses were performed with R V.3.5.2.

\section{RESULT}

\section{The characteristics of the included studies}

A total of 1102 studies were retrieved in this meta-analysis, and 279 studies were selected for full-text review. In total, 23 studies with 2068 patients fulfilled the inclusion and exclusion criteria, with publication dates ranging from 2017 to $2018 .{ }^{15-37}$ The flow chart of this study is shown in figure 1 . The sample size was between 19 and 201. Of these studies, nine were conducted in Europe,${ }^{1617212427303536}$ five were conducted in America 2228313337 and the remaining studies were conducted in Asia. Among all patients included, $1305(64.0 \%)$ were men and 643 (31.4\%) were diagnosed with squamous cell carcinoma (SCC). Twenty studies explored the association between the NLR and OS; 15 studies investigated the relationship between the NLR and PFS. Additionally, 7 of 23 studies provided data on the post-treatment NLR. ${ }^{21} 232528293233$ If the study provided data about post-treatment NLR and OS, we treated it as an independent study in the subsequent 


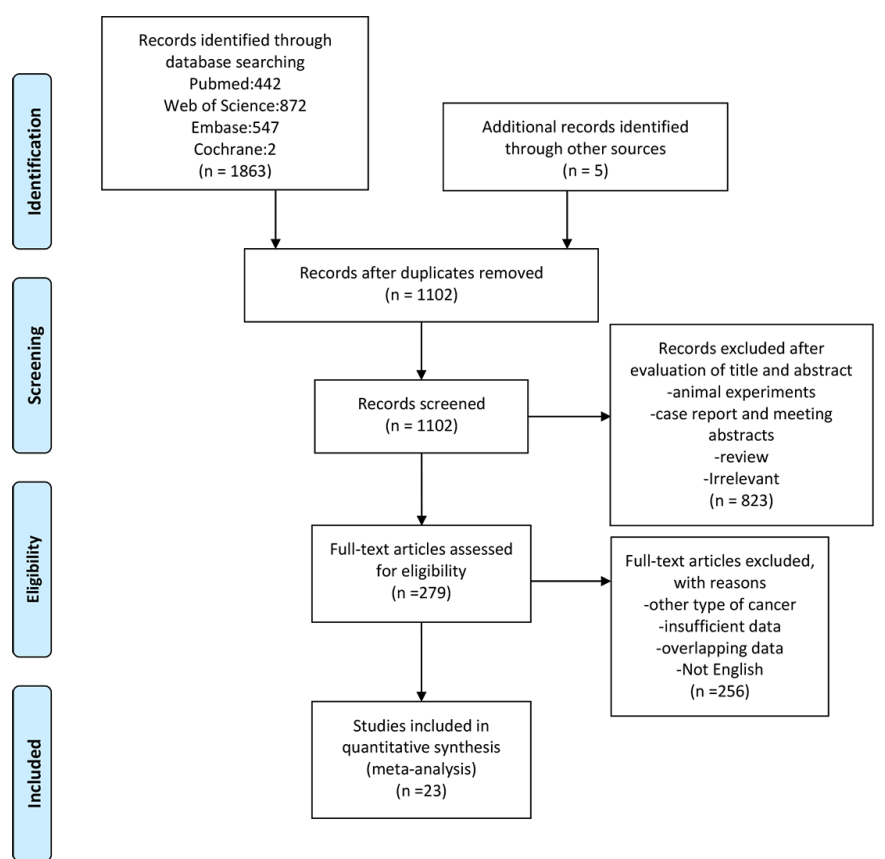

Figure 1 Flow chart of study selection.

analysis. Six trials performed first-line therapy, ${ }^{16} 1925283136$ and the other trails performed second or additional-lines of therapy. Most patients received nivolumab, a PD-1 inhibitor, as immunotherapy. The cut-off value of the NLR was not the same in all studies; a value of 5 was used frequently, and the median cut-off value for all enrolled publications was also 5. The NOS scores of the enrolled studies ranged from 6 to 9 . Detailed information on these studies is presented in table 1.

\section{Relationship between the NLR and OS in patients with lung cancer receiving immunotherapy}

Twenty studies on a total of 1629 patients treated with immunotherapy provided the NLR value or data that could be used to calculate the NLR and OS. Five of these studies provided data on the post-treatment NLR and OS. Data from a total of 23 studies were used to combine HRs and $95 \%$ CIs. In the pooled analysis of the NLR and OS, we found that a higher NLR was associated with poorer OS, with high heterogeneity $(\mathrm{HR}=1.62 ; 95 \% \mathrm{CI}: 1.41$ to 1.87; $\mathrm{p}<0.001)\left(\mathrm{I}^{2}=81.7 \%, \mathrm{p}<0.001\right)$ (figure 2$)$. To detect the source of heterogeneity, we conducted a subgroup analysis on certain clinical factors that may influence the final results, such as study design, the time at which the NLR was determined, ethnicity, sex ratio, the proportion of patients with squamous cell carcinoma (SCC\%), the NLR at baseline, the treatment line, the median follow-up time, sample size and the drug given for immunotherapy (figure 3). Interestingly, the association between the pretreatment NLR and OS showed a similar trend to the pooled result ( $\mathrm{HR}=1.87$; 95\% CI: 1.46 to 2.39 ; $\mathrm{p}<0.001)$. However, the post-treatment NLR was not significantly related to the OS in patients with lung cancer $(\mathrm{HR}=1.80$; 95\% CI: 0.81 to $4.00 ; \mathrm{p}=0.111)$. However, these results were still highly heterogeneous (pretreatment:
$I^{2}=79.80 \%, p<0.001$; post-treatment: $I^{2}=83.5 \%, p<0.001$ ). Furthermore, the NLR was significantly unrelated to the OS in studies in which SCC\% or whose baseline NLR exceeded the cut-off value was greater than $50 \%$ (figure 3). The subgroup analysis stratified by ethnicity found that patients in Asia had significantly higher HR (HR=2.76; 95\% CI: 1.88 to 4.06$)$ and less heterogeneity $\left(\mathrm{I}^{2}=45.7 \%, \mathrm{p}=0.091\right)$ than those in Europe and America $\left(\mathrm{p}_{\text {interaction }}=0.030\right)($ figure 3$)$.

\section{Relationship between the NLR and PFS in patients with lung cancer receiving immunotherapy}

Data on the NLR and PFS of 1612 patients treated with immunotherapy in 20 studies were extracted to obtain the pooled HR and 95\% CI. Four of these studies provided the post-treatment NLR and its relationship with PFS. The random effects model revealed a significant association between an elevated NLR and PFS in patients with lung cancer receiving immunotherapy $(\mathrm{HR}=1.47$; 95\% CI: 1.25 to $1.72 ; \mathrm{p}<0.001)$ with high heterogeneity $\left(\mathrm{I}^{2}=72.5 \%, \mathrm{p}<0.001\right)$ (figure 4$)$. To detect the potential source of heterogeneity in studies reporting PFS data, a subgroup analysis stratified by the factors that affect the NLR was performed as previously described (figure 5). Similar to the relationship between the NLR and OS, the NLR was significantly unrelated to the PFS in studies in which SCC\% was greater than $50 \%\left(\mathrm{p}_{\text {interaction }}=0.005\right)$. However, the pooled results for subgroups based on other factors were not markedly changed with a low level of heterogeneity.

\section{Sensitivity analysis and publication bias}

We found high heterogeneity among studies in which the relationship between the pretreatment NLR, OS and PFS was analysed. Therefore, we performed a sensitivity analysis on all enrolled studies. The effect of each study set on the combined HRs was evaluated by excluding each study individually from the meta-analysis. The results of the sensitivity analysis showed that the pooled HRs for OS and PFS were robust in our meta-analysis (figure 6A,B). We also conducted a subgroup analysis stratified by various factors to detect the source of heterogeneity. Begg's test presented no evidence of obvious publication bias in studies reporting the association between the NLR and OS $(\mathrm{p}=0.673)$ or in those reporting the association between the NLR and PFS ( $\mathrm{p}=0.074)$, but Egger's test showed significant publication bias in which both were reported ( $\mathrm{p}<0.001$ for both). Therefore, we performed a trim and fill analysis on studies reporting the relationship between the NLR and OS/PFS. However, the result was unchanged after eliminating the influence of publication bias (OS: HR=1.40; 95\% CI: 1.22 to $1.60 ; \mathrm{p}<0.001$, PFS: $\mathrm{HR}=1.33$; $95 \%$ CI: 1.14 to 1.56 ; $\mathrm{p}<0.001$, online supplementary figure 1$)$.

\section{DISCUSSION}

The results of our meta-analysis revealed the prognostic effect of both the pretreatment and post-treatment NLR 
Table 1 The basic characteristics of the enrolled studies

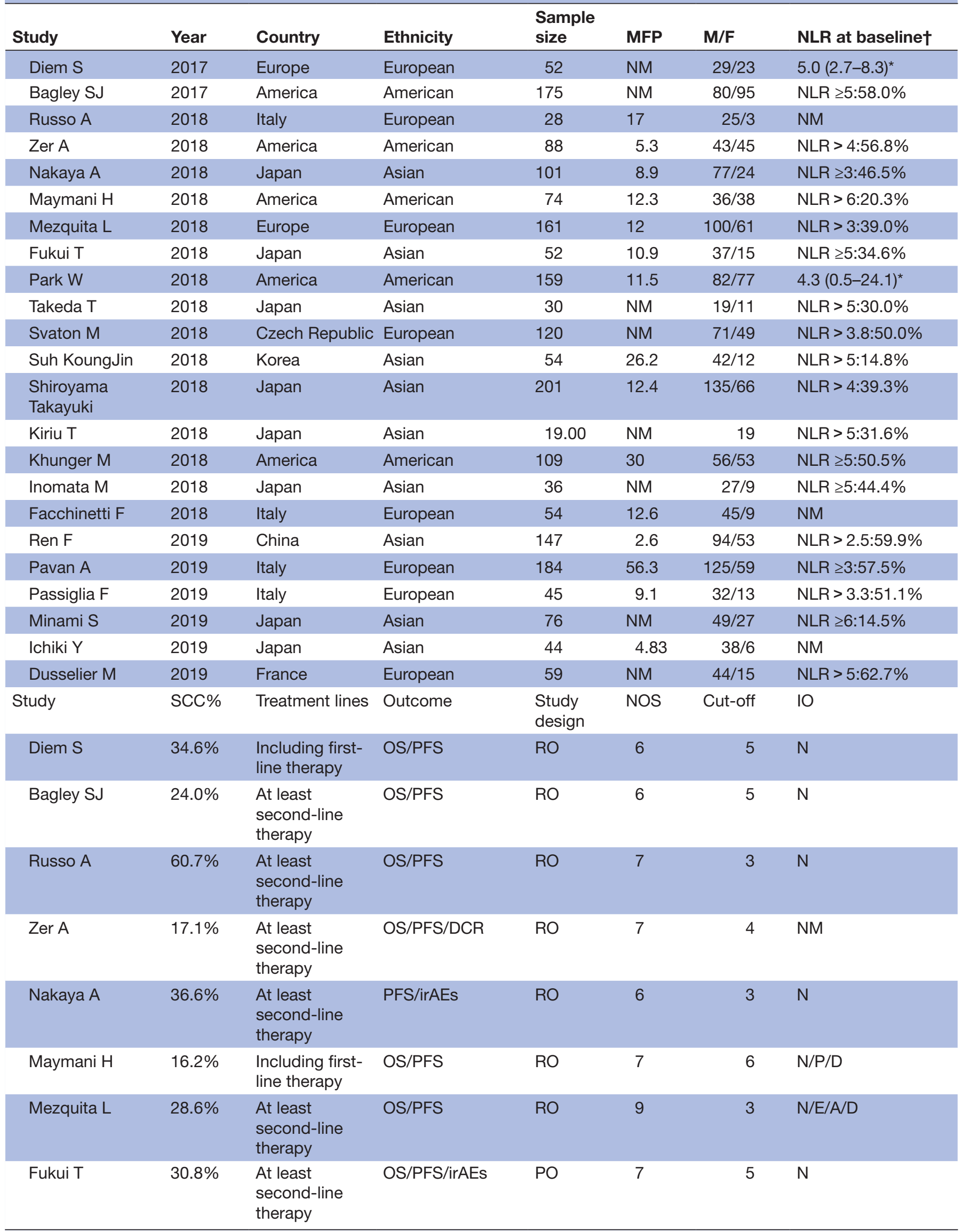




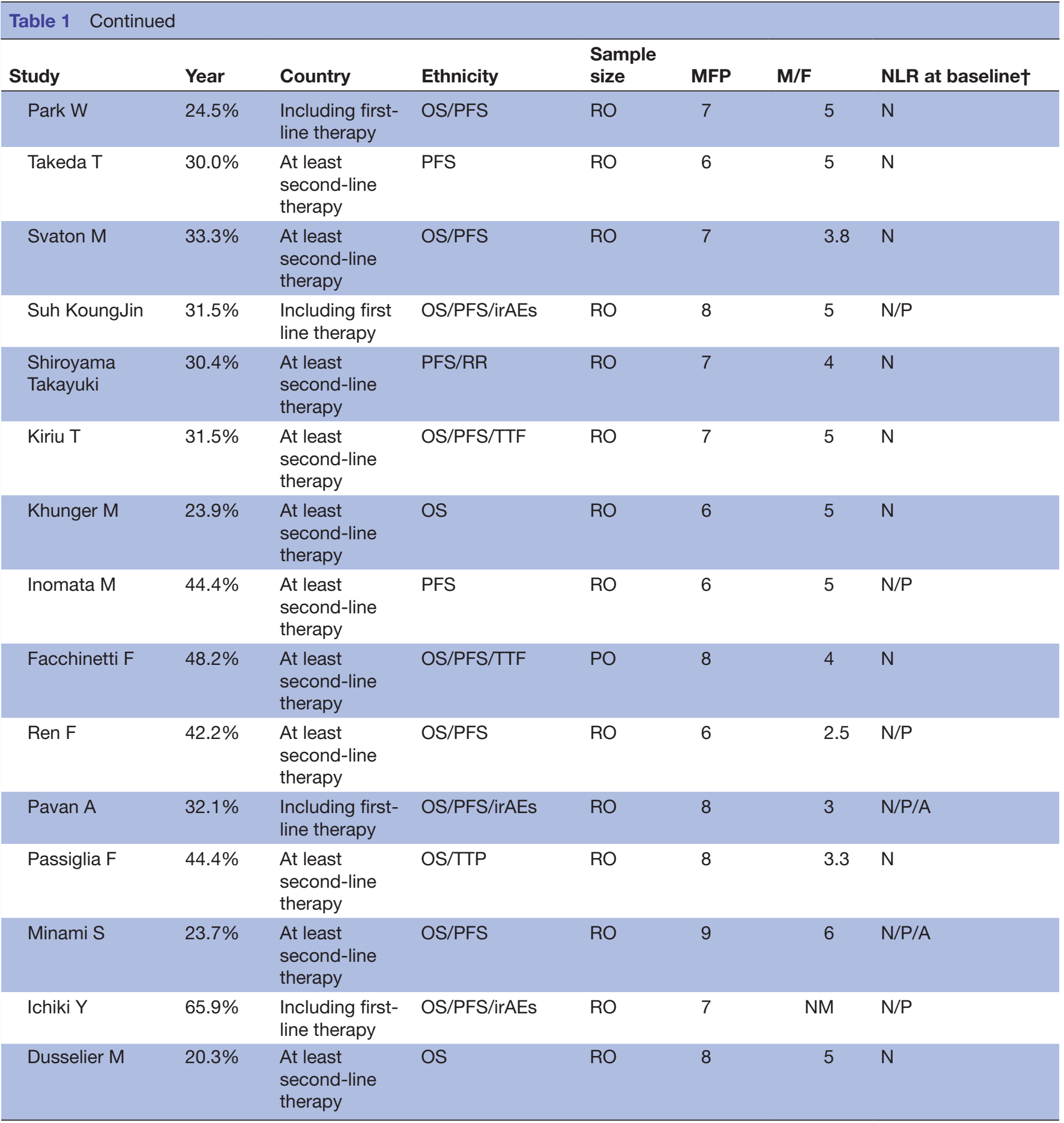

*The study provided only the median NLR and range at baseline.

$\dagger$ The proportion of the patients whose baseline NLR exceeded the cut-off value was provided

A, atezolizumab; D, durvalumab; DCR, disease control rate; E, embrolizumab; IO, immunotherapy; irAEs, immune-related adverse events; M/F, male/female; MFP, median follow-up (months); N, nivolumab; NLR, neutrophil to lymphocyte ratio; NM, not mentioned; NOS, NewcastleOttawa quality assessment Scale; OS, overall survival; P, pembrolizumab; PFS, progression-free survival; PO, prospective study; RO, retrospective study; RR, response rate; SCC\%, proportion of patients with squamous cell carcinoma; TTF, time to treatment failure; TTP, time to progression.

on OS and PFS in patients with lung cancer receiving immunotherapy. Twenty-three studies showed that an increased NLR was significantly associated with poor OS and PFS. Interestingly, the post-treatment NLR was not significantly associated with OS, and patients in Asia had significantly higher HRs than those in Europe and America. 


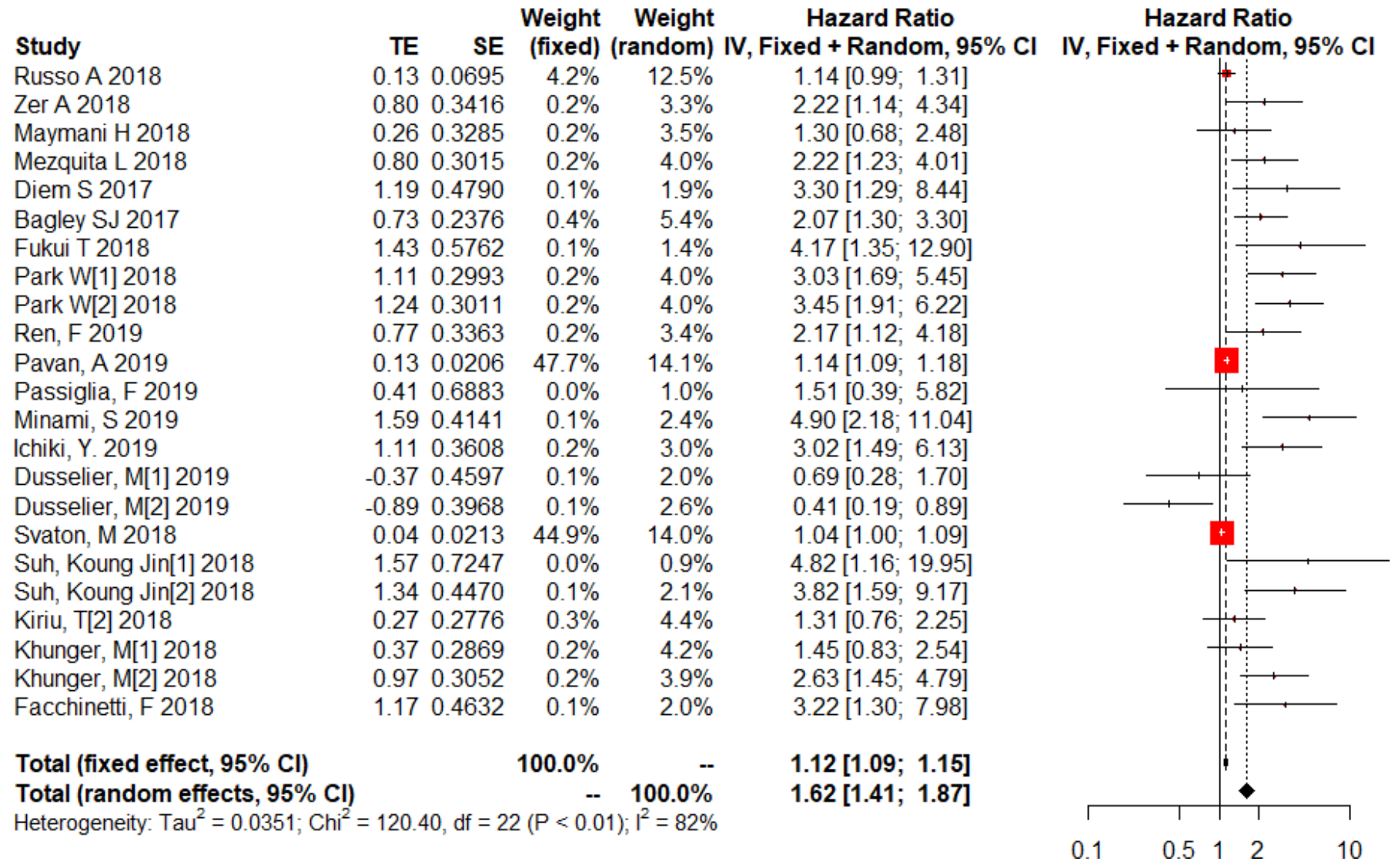

Figure 2 Forest plot of the association between the neutrophil to lymphocyte ratio and overall survival in patients with lung cancer receiving immunotherapy.

The immune checkpoint is a kind of mechanism that plays a protective role in the human immune system and acts as a brake to prevent inflammatory damage caused by the excessive activation of T cells. ${ }^{38}$ Human anti-PD-1 IgG4 $\mathrm{mAb}$ is now widely used and shows higher efficacy than standard therapies in lung cancer therapies. ${ }^{39}$ Despite a wide consensus on testing tumour tissues for PD-L1 expression, the human anti-PD-1 IgG4 mAb is limited by its 'unperfected dichotomy' across studies and molecules; patients with low levels of PD-L1 expression have response rates of up to $17 \%$, and roughly half of patients are 'not-responders' despite having high tumour levels of PD-L1. Several factors could affect the response and survival of patients receiving immunotherapy. ${ }^{39} \mathrm{In}$ addition to tumour mutation loads and the expression of tumour antigens, the status of systemic inflammation also plays an important role in patients with lung cancer receiving immunotherapy. Tumor-associated cytokines and the relevant signalling pathways could be reflected by the level of systemic inflammation, which has been proven to be associated with poor survival in patients with solid tumours. ${ }^{8}$ Biomarkers such as NLR, PLR, GPS and modified GPS have been used as prognostic factors in lung cancer. ${ }^{9-11}$ In addition, the role of systemic inflammation in patients receiving immunotherapy is particularly important for their survival. Several studies have explored the effect of the pretreatment NLR onpatients with lung cancer receiving immunotherapy. ${ }^{20} 22$ 28-31 40 There are also two meta-analyses concerning the pretreatment NLR and survival in patients with advanced cancer. ${ }^{414}$ In summary, the NLR is a reliable prognostic factor for patients with various cancer types.

Sacdalan et al reported that a high NLR resulted in poor PFS in patients with several kinds of cancers, such as melanoma, non-small-cell lung cancer (NSCLC) and genitourinary cancer, ${ }^{41}$ which was consistent with our results. However, only three publications on lung cancer were enrolled in the previous meta-analysis, and a non-significant association was discovered between the pretreatment NLR and OS was discovered. In addition, two of the three studies included in the meta-analysis previously mentioned only provided only abstracts, and we could not obtain more details about those cohorts or study designs. Another meta-analysis conducted by Jiang $\mathrm{T}$ also revealed a trend similar to ours, but the results of the subgroup analysis showed that post-treatment NLR was significantly associated with poor OS and PFS, which is in consistent with our result. Different with the study mentioned before, we enrolled more research articles and performed subgroup analyses stratified by additional clinical factors. Furthermore, our results showed that the ethnicity, the NLR at baseline and SCC\% may affect the prognostic value of the NLR. However, due to the high heterogeneity, the results must be interpreted with caution. We also found that patients in Asia had a significant higher HR than those in Europe and America 


\begin{tabular}{|c|c|c|c|c|c|c|}
\hline Subgroup & No. of Patients & No. of Studies & Hazard Ratio $(95 \% \mathrm{Cl})$ & & $1^{2}$ & $\mathrm{P}$ Value \\
\hline Overall & 1629 & 23 & $t^{10-1}$ & $1.62(1.41-1.87)$ & $81.70 \%$ & \\
\hline Cut-off value & & & & & & 0.095 \\
\hline$\geq 5$ & 834 & 14 & $\longmapsto$ & $2.08(1.42-3.04)$ & $70.20 \%$ & \\
\hline$<5$ & 751 & 8 & $\mapsto$ & $1.46(1.11-1.93)$ & $75.10 \%$ & \\
\hline Not reported & 44 & 1 & $\longmapsto$ & $3.02(1.49-6.13)$ & - & \\
\hline Study design & & & & & & 0.079 \\
\hline Retrospective & 1523 & 21 & $\longmapsto$ & $1.81(1.40-2.35)$ & $81.80 \%$ & \\
\hline Prospective & 106 & 2 & $\longmapsto$ & $3.56(1.76-7.23)$ & $0.00 \%$ & \\
\hline Time of detection & & & & & & 0.932 \\
\hline Pre-treatment & 1610 & 18 & $\mapsto-$ & $1.87(1.46-2.41)$ & $79.80 \%$ & \\
\hline Post-treatment & 19 & 5 & $\longrightarrow$ & $1.80(0.81-4.00)$ & $83.50 \%$ & \\
\hline Ethnicity & & & & & & $0.030 *$ \\
\hline European and American & 1232 & 16 & $\mapsto$ & $1.63(1.22-2.16)$ & $75.20 \%$ & \\
\hline Asian & 397 & 7 & $\longmapsto$ & $2.76(1.88-4.06)$ & $45.70 \%$ & \\
\hline Gender (M/F) & & & & & & 0.895 \\
\hline$<1$ & 337 & 3 & $\longmapsto$ & $1.86(1.34-2.59)$ & $0.00 \%$ & \\
\hline$>1$ & 1292 & 20 & $\longmapsto$ & $1.92(1.44-2.56)$ & $82.60 \%$ & \\
\hline SCC $\%$ & & & & & & 0.102 \\
\hline$>50 \%$ & 131 & 4 & $\square$ & $1.02(0.45-2.31)$ & $73.20 \%$ & \\
\hline$<50 \%$ & 1498 & 19 & $\mapsto$ & $2.07(1.65-2.58)$ & $82.90 \%$ & \\
\hline NLR at baseline $\%$ & & & & & & $0.043 *$ \\
\hline$\geqslant 50 \%$ & 851 & 10 & $\rightarrow$ & $1.36(0.97-1.92)$ & $77.80 \%$ & \\
\hline$<50 \%$ & 441 & 7 & $\longmapsto$ & $2.47(1.59-3.84)$ & $56.30 \%$ & \\
\hline Not reported & 337 & 6 & $\longmapsto$ & $2.42(1.61-3.62)$ & $85.70 \%$ & \\
\hline Treatment line & & & & & & 0.167 \\
\hline At least second line therapy & 1057 & 15 & $\mapsto$ & $1.67(1.22-2.29)$ & $79.00 \%$ & \\
\hline Including first line therapy & 572 & 8 & $\longmapsto$ & $2.36(1.62-3.44)$ & $85.20 \%$ & \\
\hline Median follow-up & & & & & & $0.043 *$ \\
\hline$\geqslant 12$ & 669 & 9 & $\longmapsto$ & $1.78(1.28-2.46)$ & $72.70 \%$ & \\
\hline$<12$ & 535 & 7 & $\longmapsto$ & $2.77(2.11-3.63)$ & $0.00 \%$ & \\
\hline Not reported & 425 & 7 & $\longrightarrow$ & $1.42(0.76-2.66)$ & $82.80 \%$ & \\
\hline Sample Size & & & & & & 0.085 \\
\hline$\geq 100$ & 631 & 9 & $\longmapsto$ & $1.84(1.37-2.45)$ & $86.70 \%$ & \\
\hline$<100$ & 998 & 14 & $\longmapsto$ & $1.93(1.30-2.86)$ & $75.40 \%$ & \\
\hline ICI & & & & & & 0.294 \\
\hline $\mathrm{N}$ & 786 & 14 & $\longmapsto$ & $1.69(1.20-2.38)$ & $81.70 \%$ & \\
\hline N/P & 250 & 4 & $\longmapsto$ & $2.91(1.94-4.31)$ & $0.00 \%$ & \\
\hline N/P/A & 260 & 2 & . & $2.23(0.53-9.28)$ & $91.90 \%$ & \\
\hline N/P/D & 74 & 1 & . & $1.30(0.68-2.48)$ & - & \\
\hline N/E/AND & 161 & 1 & $\longmapsto$ & $2.22(1.23-4.01)$ & - & \\
\hline Not reported & 88 & 1 & $\longmapsto$ & $2.22(1.14-4.34)$ & - & \\
\hline
\end{tabular}

Figure 3 Subgroup analysis of the relationship between the neutrophil to lymphocyte ratio (NLR) and overall survival in patients with lung cancer receiving immunotherapy. *The data here show the proportion of patients whose baseline NLR exceeded the cut-off value.A, atezolizumab;D, durvalumab; E, embrolizumab; ICI, immune checkpoint inhibitor; M/F, male/ female; $N$, nivolumab; $\mathrm{P}$, pembrolizumab;SCC\%, proportion of patients with squamous cell carcinoma.

\section{Study}

Russo A 2018

Zer A 2018

Nakaya A[1] 2018

Nakaya A[2] 2019

Mezquita L 2018

Bagley SJ 2017

Fukui T 2018

Park W[1] 2018

Park W[2] 2018

Ren, F 2019

Pavan, A 2019

Minami, S 2019

Takeda, T[1] 2018

Takeda, T[2] 2018

Takeda, T[3] 2018

Svaton, M 2018

Suh, Koung Jin[1] 2018

Suh, Koung Jin[2] 2018

Shiroyama, Takayuki 2018

Inomata, M 2018
TE SE

0.050 .0839

$\begin{array}{lll}0.54 & 0.2777\end{array}$

0.190 .2438

0.310 .2996

$0.60 \quad 0.2496$

$0.36 \quad 0.1718$

$0.72 \quad 0.3169$

$\begin{array}{lll}0.52 & 0.2132\end{array}$

$\begin{array}{lll}0.60 & 0.2092\end{array}$

0.320 .1688

0.590 .1974

0.450 .3544

0.200 .7033

$\begin{array}{lll}-0.63 & 0.4970\end{array}$

1.790 .8103

$0.03 \quad 0.0242$

0.750 .5005

2.710 .6118

$0.38 \quad 0.1620$

$\begin{array}{lll}0.09 & 0.2357\end{array}$
Weight Weight

$6.7 \% \quad 9.2 \%$

$0.6 \% \quad 4.7 \%$

$0.8 \% \quad 5.4 \%$

$0.5 \% \quad 4.3 \%$

$0.8 \% \quad 5.2 \%$

$1.6 \% \quad 7.0 \%$

$0.5 \% \quad 4.0 \%$

$1.0 \% \quad 6.0 \%$

$1.1 \% \quad 6.1 \%$

$1.6 \% \quad 7.1 \%$

$1.2 \% \quad 6.4 \%$

$0.4 \% \quad 3.5 \%$

$0.1 \% \quad 1.2 \%$

$0.2 \% \quad 2.2 \%$

$0.1 \% \quad 0.9 \%$

$80.0 \% \quad 10.1 \%$

$0.2 \% \quad 2.1 \%$

$0.1 \% \quad 1.5 \%$

$1.8 \% \quad 7.3 \%$

$0.8 \% \quad 5.5 \%$

Total (fixed effect, $95 \% \mathrm{Cl}$ )

Total (random effects, $95 \% \mathrm{Cl}$ )

\section{$100.0 \% \quad-7$ \\ $-100.0 \%$}

Heterogeneity: $\mathrm{Tau}^{2}=0.0661 ; \mathrm{Chi}^{2}=68.98, \mathrm{df}=19(\mathrm{P}<0.01) ; \mathrm{I}^{2}=72 \%$

Hazard Ratio Hazard Ratio

$1.05[0.89 ; 1.24]$

$1.72[1.00 ; 2.96]$

$1.21[0.75 ; 1.95]$

$1.37[0.76 ; 2.46]$

$1.83[1.12 ; 2.99]$

$1.43[1.02 ; 2.00]$

$2.05[1.10 ; 3.82]$

$1.69[1.11 ; 2.57]$

$1.82[1.21 ; 2.74]$

$1.38[0.99 ; 1.93]$

$1.80[1.22 ; 2.65]$

$1.56[0.78 ; 3.13]$

$1.23[0.31 ; 4.87]$

$0.53[0.20 ; 1.41]$

$6.00[1.22 ; 29.34]$

$1.03[0.99 ; 1.08]$

$2.11[0.79 ; 5.62]$

$15.09[4.55 ; 50.05]$

$1.46[1.06 ; 2.01]$

$1.09[0.69 ; 1.73]$

\section{IV, Fixed + Random, $95 \% \mathrm{Cl}$}

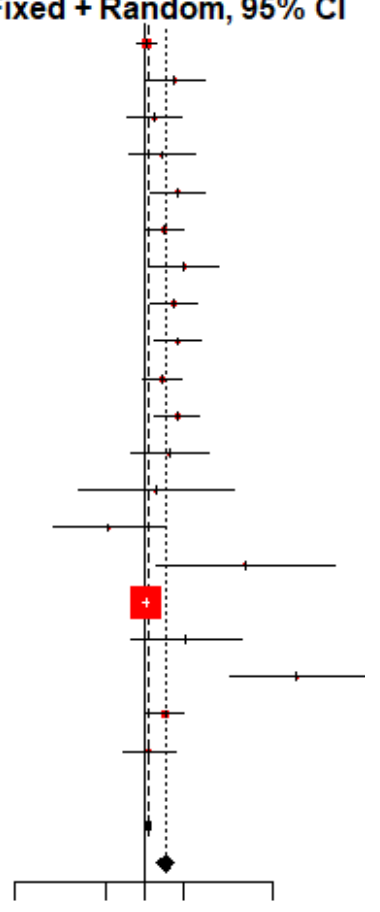

$\begin{array}{llll}0.1 & 0.51 & 2 & 10\end{array}$

Figure 4 Forest plot of the association between the neutrophil to lymphocyte ratio (NLR) and progression-free survival in patients with lung cancer receiving immunotherapy. 


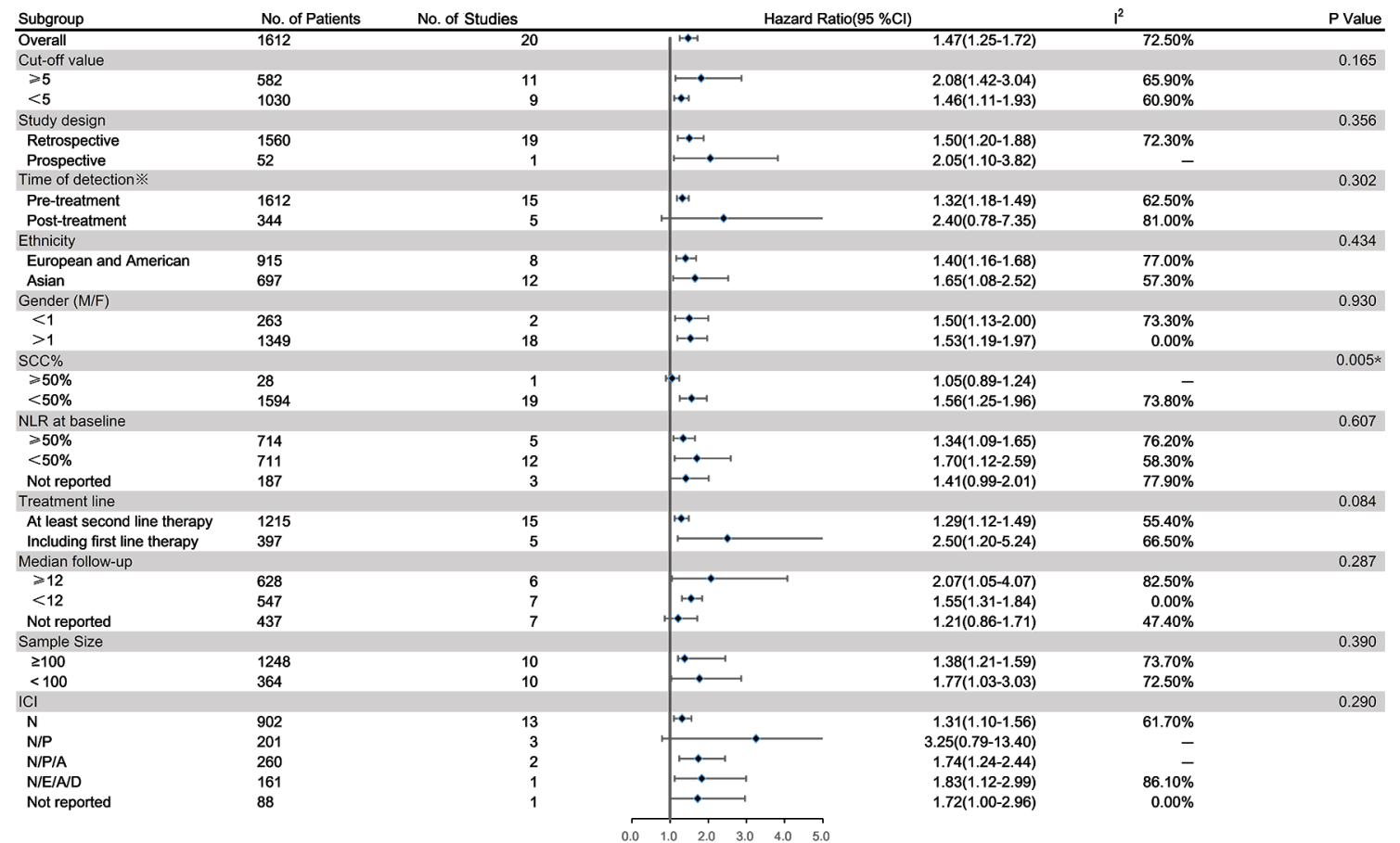

Figure 5 Subgroup analysis of the relationship between the neutrophil to lymphocyte ratio (NLR) and progression-free survival (PFS) in patients with lung cancer receiving immunotherapy. ${ }^{*}$ Twenty studies provided the data on the pretreatment NLR and PFS, and 5 of them also provided the post-treatment NLR and PFS. A, atezolizumab;D, durvalumab;E, embrolizumab;ICl, immune checkpoint inhibitor; M/F, male/female; N, nivolumab;P, pembrolizumab; SCC\%, proportion of patients with squamous cell carcinoma.

in the subgroup analysis of the relationship between the NLR and OS. Some studies showed that neutrophils were the most abundant immune cell type identified in NSCLC patients and accounted for nearly $20 \%$ of all CD45+ cells in patients from America. ${ }^{43}$ However, this result was not found in patients from Asia or Europe. The systemic inflammatory response in different ethnicities might differ. Furthermore, we collected baseline patient information, including SCC\%, from all studies, and our results showed that the histology of lung cancer might have an impact on the prognostic value of the NLR.
Many factors, including tumour mutation load and the expression of tumour antigens, affect patient response and survival. ${ }^{39}$ Patients with lung adenocarcinoma have a high epidermal growth factor receptor (EGFR) mutation rate and some studies revealed that patients with targetable oncogenes were associated with a poor response to immunotherapy. ${ }^{44}$ This may account for the results of our article.

The current study had several limitations. First, high heterogeneity was present in this analysis although we conducted sensitivity analyses on all studies. The results
A

Study
Omitting Russo A 2018
Omitting Zer A 2018
Omitting Maymani H 2018
Omitting Mezquita L 2018
Omitting Diem S 2017
Omiting Bagley SJ 2017
Omitting Fukui T 2018
Omitting Park W[1] 2018
Omitting Park W[2] 2018
Omitting Ren, F 2019
Omitting Pavan, A 2019
Omitting Passiglia, F 2019
Omitting Minami, S 2019
Omitting Ichiki, Y 2019
Omitting Dusselier, M[1] 2019
Omitting Dusselier, M[2] 2019
Omitting Svaton, M 2018
Omitting Suh, Koung Jin[1] 2018
Omitting Suh, Koung Jin[2] 2018
Omitting Kiriu, T[2] 2018
Omitting Khunger, M[1] 2018
Omitting Khunger, M[2] 2018
Omitting Facchinetti, F 2018
Random effects model
Random

Study

Omitting Maymani $\mathrm{H} 201$

Omitting Mezquita L 2018

Omitting Bagley SJ 2017

Omitting Fukui T 2018

Omitting Park W[1] 2018

Omitting Ren, F 2019

Omitting Passiglia, $\mathrm{F} 2019$

Omitting Minami, S 2019

Omitting Dusselier, M[1]2019

Omitting Dusselier, M[2] 2019

Omitting Suh, Koung Jin[1]2018

Omitting Suh, Koung Jin[2] 2018

Random effects model

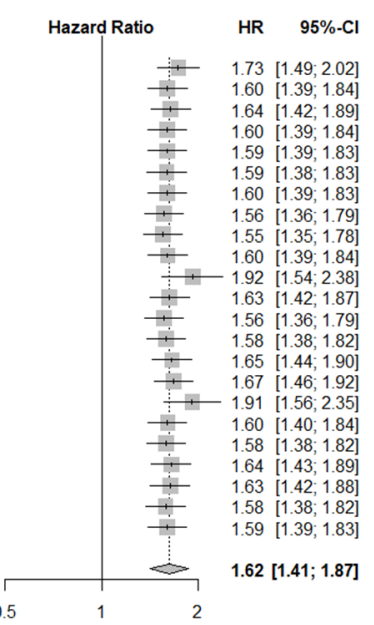

B

Study

Omitting Russo A 2018 Omitting Zer A 2018 Omitting Nakaya A[1] 2018 Omitting Nakaya A[2] 2019 Omitting Mezquita L 2018 Omitting Bagley SJ 2017

Omitting Fukui T 2018

Omitting Park W[1] 2018

Omitting Park W[2] 2018

Omitting Ren, F 2019

Omitting Pavan, A 2019

Omitting Minami, S 2019

Omitting Takeda, T[1] 2018

Omitting Takeda, T[2] 2018

Omitting Takeda, T[3] 2018

Omitting Suh, Koung Jin[1] 2018

Omitting Suh, Koung Jin[2] 2018

Omitting Shiroyama, Takayuki 2018

Omitting Inomata, M 2018

Random effects model

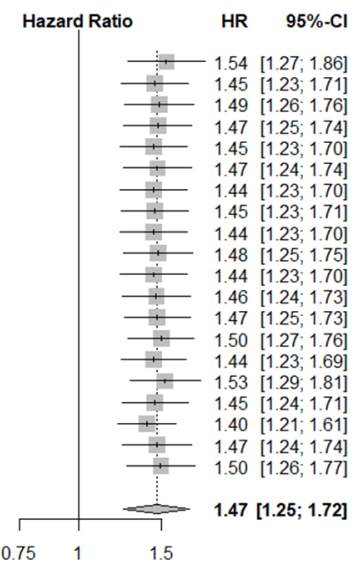

Figure 6 Sensitivity analysis of overall survival (A) and progression-free survival (B). 
were robust after eliminating each study from the analysis. In addition, we performed subgroup analyses on certain possible impact factors to detect the source of heterogeneity. Second, Egger's test showed that obvious publication bias in the current study. The pooled results should be treated with caution, although trim and fill analysis testing indicated credibility for this study. Additionally, considering the high heterogeneity after subgroup analysis, other factors might be responsible for the high heterogeneity in this meta-analysis.

\section{CONCLUSION}

Generally, our meta-analysis focused on the clinical prognostic agreement of the NLR and OS and PFS in patients with lung cancer. Importantly, given the limitations mentioned above, these findings should be treated with caution in clinical practice. More prospective cohort studies are needed to confirm our results.

Acknowledgements This work was supported by the Transformation Projects of Sci-Tech Achievements of Sichuan Province (2016CZYD0001), the Sci-Tech Support Program of Science and Technology Department of Sichuan Province (2016SZ0073), the National Major Sci-Tech Project (2017Z×10103004-012) and the National Key Development Plan for Precision Medicine Research (2017YFC0910004)

Contributors Conception and design: WL, JJ and LY; Administrative support: JJ and WL; Provision of study materials or patients: DL; Collection and assembly of data: JJ and DL; Data analysis and interpretation: DL and LY; Manuscript writing: all authors; Final approval of manuscript: all authors.

Funding The authors have not declared a specific grant for this research from any funding agency in the public, commercial or not-for-profit sectors.

Competing interests None declared.

Patient consent for publication Not required.

Ethics approval All procedures performed in the studies involving human participants were in accordance with the ethical standards of the institutional and/ or national research committee and with the 1964 Helsinki Declaration and its later amendment.

Provenance and peer review Not commissioned; externally peer reviewed.

Data availability statement Data are available in a public, open access repository. all data in this article are available from published articles.

Open access This is an open access article distributed in accordance with the Creative Commons Attribution Non Commercial (CC BY-NC 4.0) license, which permits others to distribute, remix, adapt, build upon this work non-commercially, and license their derivative works on different terms, provided the original work is properly cited, appropriate credit is given, any changes made indicated, and the use is non-commercial. See: http://creativecommons.org/licenses/by-nc/4.0/.

\section{ORCID iD}

Weimin Li http://orcid.org/0000-0003-2859-8668

\section{REFERENCES}

1 Siegel RL, Miller KD, Jemal A. Cancer statistics, 2015. CA Cancer J Clin 2015;65:5-29.

2 Callahan MK, Postow MA, Wolchok JD. Targeting T cell co-receptors for cancer therapy. Immunity 2016;44:1069-78.

3 Chen Y-M. Immune checkpoint inhibitors for nonsmall cell lung cancer treatment. J Chin Med Assoc 2017;80:7-14.

4 Yarchoan M, Hopkins A, Jaffee EM. Tumor mutational burden and response rate to PD-1 inhibition. N Engl J Med 2017;377:2500-1.

5 Anagnostou V, Smith KN, Forde PM, et al. Evolution of neoantigen landscape during immune checkpoint blockade in non-small cell lung cancer. Cancer Discov 2017;7:264-76.
6 Goswami S, Basu S, Sharma P. A potential biomarker for anti-PD-1 immunotherapy. Nat Med 2018;24:123-4.

7 Marchetti A, Barberis M, Franco R, et al. Multicenter comparison of 22C3 PharmDx (Agilent) and SP263 (Ventana) assays to test PD-L1 expression for NSCLC patients to be treated with immune checkpoint inhibitors. J Thorac Oncol 2017;12:1654-63.

8 Hooper CE, Lyburn ID, Searle J, et al. The South West area mesothelioma and pemetrexed trial: a multicentre prospective observational study evaluating novel markers of chemotherapy response and prognostication. Br J Cancer 2015;112:1175-82.

9 Wang X, Teng F, Kong L, et al. Pretreatment neutrophil-to-lymphocyte ratio as a survival predictor for small-cell lung cancer. Onco Targets Ther 2016;9:5761-70.

10 Sanchez-Salcedo P, de-Torres JP, Martinez-Urbistondo D, et al. The neutrophil to lymphocyte and platelet to lymphocyte ratios as biomarkers for lung cancer development. Lung Cancer 2016;97:28-34.

11 Kos M, Hocazade C, Kos FT, et al. Prognostic role of pretreatment platelet/lymphocyte ratio in patients with non-small cell lung cancer. Wien Klin Wochenschr 2016;128:635-40.

12 Tierney JF, Stewart LA, Ghersi D, et al. Practical methods for incorporating summary time-to-event data into meta-analysis. Trials 2007;8:16.

13 Begg CB, Mazumdar M. Operating characteristics of a RANK correlation test for publication bias. Biometrics 1994;50:1088

14 O'Rourke K, Shea B, Wells GA. Meta-Analysis of clinical trials. New York: Springer, 2001: 397-424.

15 Ren F, Zhao T, Liu B, et al. Neutrophil-Lymphocyte ratio (NLR) predicted prognosis for advanced non-small-cell lung cancer (NSCLC) patients who received immune checkpoint blockade (ICB). Onco Targets Ther 2019;12:4235-44.

16 Pavan A, Calvetti L, Dal Maso A, et al. Peripheral blood markers identify risk of immune-related toxicity in advanced non-small cell lung cancer treated with Immune-Checkpoint inhibitors. Oncologist 2019;24:1128-36.

17 Passiglia F, Galvano A, Castiglia M, et al. Monitoring blood biomarkers to predict nivolumab effectiveness in NSCLC patients. Ther Adv Med Oncol 2019;11:1758835919839928.

18 Minami S, Ihara S, Ikuta S, et al. Gustave Roussy immune score and Royal Marsden Hospital prognostic score are biomarkers of ImmuneCheckpoint inhibitor for non-small cell lung cancer. World J Oncol 2019;10:90-100.

19 Ichiki Y, Taira A, Chikaishi Y, et al. Prognostic factors of advanced or postoperative recurrent non-small cell lung cancer targeted with immune check point inhibitors. J Thorac Dis 2019;11:1117-23.

20 Fukui T, Okuma Y, Nakahara $Y$, et al. Activity of nivolumab and utility of neutrophil-to-lymphocyte ratio as a predictive biomarker for advanced non-small-cell lung cancer: a prospective observational study. Clin Lung Cancer 2019;20:208-14.

21 Dusselier M, Deluche E, Delacourt N, et al. Neutrophil-ToLymphocyte ratio evolution is an independent predictor of early progression of second-line nivolumab-treated patients with advanced non-small-cell lung cancers. PLoS One 2019;14:e0219060.

22 Zer A, Sung MR, Walia P, et al. Correlation of neutrophil to lymphocyte ratio and absolute neutrophil count with outcomes with PD-1 axis inhibitors in patients with advanced non-small-cell lung cancer. Clin Lung Cancer 2018;19:426-34.

23 Takeda T, Takeuchi M, Saitoh M, et al. Neutrophil-To-Lymphocyte ratio after four weeks of nivolumab administration as a predictive marker in patients with pretreated non-small-cell lung cancer. Thorac Cancer 2018;9:1291-9.

24 Svaton M, Zemanova M, Skrickova J, et al. Chronic inflammation as a potential predictive factor of nivolumab therapy in non-small cell lung cancer. Anticancer Res 2018;38:6771-82.

25 Suh KJ, Kim SH, Kim YJ, et al. Post-Treatment neutrophil-tolymphocyte ratio at week 6 is prognostic in patients with advanced non-small cell lung cancers treated with anti-PD-1 antibody. Cancer Immunol Immunother 2018;67:459-70.

26 Shiroyama T, Suzuki H, Tamiya M, et al. Pretreatment advanced lung cancer inflammation index (Ali) for predicting early progression in nivolumab-treated patients with advanced non-small cell lung cancer. Cancer Med 2018;7:13-20.

27 Russo A, Franchina T, Ricciardi GRR, et al. Baseline neutrophilia, derived neutrophil-to-lymphocyte ratio (dNLR), platelet-tolymphocyte ratio (PLR), and outcome in non small cell lung cancer (NSCLC) treated with nivolumab or docetaxel. J Cell Physiol 2018;233:6337-43.

28 Park W, Kwon D, Saravia D, et al. Developing a predictive model for clinical outcomes of advanced non-small cell lung cancer patients treated with nivolumab. Clin Lung Cancer 2018;19:280-8. 
29 Nakaya A, Kurata T, Yoshioka H, et al. Neutrophil-To-Lymphocyte ratio as an early marker of outcomes in patients with advanced non-small-cell lung cancer treated with nivolumab. Int J Clin Oncol 2018;23:634-40.

30 Mezquita L, Auclin E, Ferrara R, et al. Association of the lung immune prognostic index with immune checkpoint inhibitor outcomes in patients with advanced non-small cell lung cancer. JAMA Oncol 2018;4:351-7.

31 Maymani H, Hess K, Groisberg R, et al. Predicting outcomes in patients with advanced non-small cell lung cancer enrolled in early phase immunotherapy trials. Lung Cancer 2018;120:137-41.

32 Kiriu T, Yamamoto M, Nagano T, et al. The time-series behavior of neutrophil-to-lymphocyte ratio is useful as a predictive marker in non-small cell lung cancer. PLoS One 2018;13:e0193018.

33 Khunger M, Patil PD, Khunger A, et al. Post-Treatment changes in hematological parameters predict response to nivolumab monotherapy in non-small cell lung cancer patients. PLoS One 2018;13:e0197743.

34 Inomata M, Hirai T, Seto Z, et al. Clinical parameters for predicting the survival in patients with squamous and Non-squamouscell NSCLC receiving PD-1 inhibitor therapy. Pathol Oncol Res 2020;26:327-33.

35 Facchinetti F, Veneziani M, Buti S, et al. Clinical and hematologic parameters address the outcomes of non-small-cell lung cancer patients treated with nivolumab. Immunotherapy 2018;10:681-94.

36 Diem S, Schmid S, Krapf M, et al. Neutrophil-To-Lymphocyte ratio (NLR) and platelet-to-lymphocyte ratio (PLR) as prognostic markers in patients with non-small cell lung cancer (NSCLC) treated with nivolumab. Lung Cancer 2017;111:176-81.
37 Bagley SJ, Kothari S, Aggarwal C, et al. Pretreatment neutrophilto-lymphocyte ratio as a marker of outcomes in nivolumab-treated patients with advanced non-small-cell lung cancer. Lung Cancer 2017;106:1-7.

38 Ito A, Kondo S, Tada K, et al. Clinical development of immune checkpoint inhibitors. Biomed Res Int 2015;2015:605478

39 Carbone DP, Reck M, Paz-Ares L, et al. First-Line nivolumab in stage IV or recurrent non-small-cell lung cancer. $N$ Engl J Med 2017;376:2415-26.

40 Russo A, Franchina T, Ricciardi GRR, et al. Baseline neutrophilia, derived neutrophil-to-lymphocyte ratio (dNLR), platelet-tolymphocyte ratio (PLR), and outcome in non small cell lung cancer (NSCLC) treated with nivolumab or docetaxel. J Cell Physiol 2018.

41 Sacdalan DB, Lucero JA, Sacdalan DL. Prognostic utility of baseline neutrophil-to-lymphocyte ratio in patients receiving immune checkpoint inhibitors: a review and meta-analysis. Onco Targets Ther 2018;11:955-65.

42 Jiang T, Bai Y, Zhou F, et al. Clinical value of neutrophil-tolymphocyte ratio in patients with non-small-cell lung cancer treated with PD-1/PD-L1 inhibitors. Lung Cancer 2019;130:76-83.

43 Kargl J, Busch SE, Yang GHY, et al. Neutrophils dominate the immune cell composition in non-small cell lung cancer. Nat Commun 2017;8:14381.

44 Mazieres J, Drilon A, Lusque A, et al. Immune checkpoint inhibitors for patients with advanced lung cancer and oncogenic driver alterations: results from the IMMUNOTARGET registry. Ann Oncol 2019;30:1321-8. 